\title{
Smoking Cessation Treatment Programs Offered at Hospitals Providing Oncology Services
}

\author{
Michael Shayne Gallaway, Eric Tai, and Elizabeth A. Rohan \\ Division of Cancer Prevention and Control, National Center for Chronic Disease Prevention and Health Promotion, Centers for \\ Disease Control and Prevention, Atlanta, Georgia
}

\begin{abstract}
$\mathrm{B}$ ackground: Many people with cancer continue smoking despite evidence that it negatively effects cancer treatment, worsens chemotherapy toxicity, and increases risk for a second cancer. Aims: We examined tobacco treatment services offered to cancer patients at hospitals providing oncology services, including National Cancer Institute (NCl)-Designated Cancer Centers (NDCCs). Methods: We examined survey data of 6,400 U.S. hospitals from 2008 to 2015 to determine the manner in which tobacco treatment/cessation program services were provided among NDCCs and non-NDCC hospitals providing oncology services (HPOs).

Results: From 2008 to 2015, 784 responses from NDCCs and 18,281 responses from HPOs were received. NDCCs (86\%) reported significantly higher tobacco treatment/cessation programs owned by the hospital compared to HPOs $(68 \%)(p<0.001)$. Among NDCCs, there was a significant increasing trend of tobacco treatment/cessation programs reported owned by the hospital, the health system, or other contractual mechanism from 2008 to 2015 ( $p=0.03$ ).

Conclusions: More than $80 \%$ of oncology providing hospitals report providing tobacco cessation programs, with higher percentages reported in NDCCs. As hospitals implement smoking cessation programs, partnerships between hospitals and cancer coalitions could help bring tobacco cessation activities to communities they both serve, and link discharged patients to these cessation resources so they can continue quit attempts that they initialised while hospitalised.
\end{abstract}

\section{Introduction}

The prevalence of smoking among persons diagnosed with cancer is similar to the general population (Cox, Africano, Tercyak, \& Taylor, 2003; Emmons et al., 2002; Ramaswamy, Toll, Chagpar, \& Judson, 2016; Shoemaker, White, Hawkins, \& Hayes, 2016; Tyc, 2005; Underwood et al., 2012). Many people with cancer continue to use cigarettes (Gritz, 2005; Klosky et al., 2007; Mackenbach, 2001; Mariotto, 2007) despite evidence of their negative effects on cancer treatment and other outcomes (Blum, 1997; Browman et al., 1993; Day, 1994; Des Rochers, Dische, \& Saunders, 1992; Do et al., 2003; Gan et al., 2013; Khuri et al., 2006; Mason et al., 2009; Richardson, 1993; Shiels et al., 2014; Wynder, 1977). Smoking cessation treatments can be effective among this population (Fiore et al., 2008; Kawahara et al., 1998; Tucker, 1997).
Several evidence-based cessation treatments are available to assist smokers (Gritz et al., 2006; Siu, 2015).

Approximately 250,000 cancer patients are treated at National Cancer Institute (NCI)-Designated Cancer Centers (NDCCs) or directly affiliated hospitals annually (National Cancer Institute, 2017b). However, there is minimal research on tobacco treatment services offered at these facilities, and it is an area that requires further study (Singhi et al., 2015). One available study (Goldstein, Ripley-Moffitt, Pathman, \& Patsakham, 2013) reported that about half of NDCCs offered tobacco treatment services, and one-quarter had a program to refer patients (in addition to the services offered). Health People 2020 identified increasing tobacco screening efforts and tobacco cessation counselling in hospital ambulatory care settings as means to decrease tobacco use (Office of

\footnotetext{
Address for correspondence: Michael Shayne Gallaway, PhD, MPH, Division of Cancer Prevention and Control, National Center for Chronic Disease Prevention and Health Promotion, Centers for Disease Control and Prevention, 4770 Buford Highway, MS F76, Atlanta, Georgia 30341. Email: mgallaway@cdc.gov
} 
Disease Prevention and Health Promotion, 2017). In this study, we examined treatment/cessation programs administered by hospitals providing oncology services and provided a descriptive analysis of the number of tobacco cessation programs available for patients at these facilities.

\section{Methods}

We examined 2008-2015 American Hospital Association (AHA) survey (American Hospital Association, 2017b) data collected from over 6,400 U.S hospitals. It is the largest annual survey of U.S. hospitals and collects information on hospital demographics, organisational structure, service types and utilisation, and business arrangements with physicians. Survey participants are not required to be members or registered with the AHA. Nonregistered hospitals are identified through state hospital associations, the Centers for Medicare and Medicaid Services, and other national organisations and government entities. The overall annual response rate is approximately $80 \%$ (American Hospital Association, 2017b). Unusual changes in data from one year to next are validated through contact with the hospital, and historic trends are examined for inconsistencies. Hospitals' data that was imputed (due to non-response) were excluded from this study. Survey responses are supplemented by data drawn from AHA registration database, the U.S. Census Bureau, hospital accrediting bodies, and other organisations. Hospitals completed the survey annually for the preceding operational year, and as such hospitals that completed the survey each year are included in multiple years of data when examined over time (2008-2015). We decided to include all completed surveys for trend analyses to get a more accurate representation of services offered in each given operational year examined. However, only the most recent year of the data was included to summarise facility characteristics for NDCCs and HPOs.

\section{Measures}

We examined tobacco treatment/cessation programs among hospitals providing oncology services. All NDCCs and directly affiliated hospitals providing patient care were identified by designation year (National Cancer Institute, 2017b). NDCC Basic Laboratories were excluded. Hospitals were grouped into two mutually exclusive categories: (1) NDCCs and directly affiliated facilities and (2) hospitals that reported providing oncology services owned by their hospital or its subsidiary (HPOs). Hospitals not meeting either of these two definitions were excluded. Hospitals were asked how tobacco treatment/cessation services are provided, specifying one or more: 'Owned or provided by my hospital or its subsidiary', 'Provided by my Health System (in my local community)', 'Provided through a formal contractual arrangement or joint venture with another provider not in my system (in my local community)', or 'Do not provide'. Descriptions and definitions of survey items have been previously published (American Hospital Association, 2017a).

\section{Statistical Analysis}

Facility characteristics were stratified by group (NDCCs, HPOs). We analysed the data by NCI designation (Cancer Center, Comprehensive Cancer Center), hospital authority operations [Government (Federal, nonFederal), non-Government (not-for-profit), or Investorowned (for profit)], capacity, utilisation, and staffing. Chi-square and Kruskal-Wallis tests were used to assess significant differences between groups. A test for trend was calculated to assess whether tobacco treatment/cessation program services increased significantly from 2008 to 2015. SAS (SAS Institute, Cary, NC) Enterprise Guide version 7.11 was used for all analyses.

\section{Results}

From 2008 to 2015, an average of 98 NDCCs and 2,285 HPOs completed the AHA survey annually, with a total of 784 responses from NDCCs and 18,281 responses from HPOs during this timeframe. Of the 784 NDCC responses, $86.4 \%$ were from NCI-Designated Comprehensive Cancer Centers (Table 1).

The hospital authority for policy for NDCCs was significantly more likely to be Federal or non-Federal Government (98.8\%), compared to HPOs $(86.0 \%)(p<$ $0.001)$. NDCCs reported significantly more capacity, annual utilisation, and staffing compared with HPOs as reported for all hospital services. The mean number of total licensed beds reported by NDCCs (571) was more than double reported by HPOs $(277)(p<0.001)$. The mean number of total facility personnel reported annually by NDCCs $(5,553)$ was more than triple of that reported by HPOs $(1,490)(p<0.001)$. Correspondingly, utilisation (mean number of admissions, inpatient days, and outpatient visits) reported by NDCCs was more than double reported by HPOs $(p<0.001)$.

NDCCs reported a significantly higher percentage of any tobacco treatment/cessation programs owned by the hospital or its subsidiary or provided in the local community (87.6-95.9\%) than HPOs (75.1-80.3\%) (Table 2). The majority of cessation programs in both NDCCs and HPOs were owned by the hospital or its subsidiary $(82.4 \%$ and $69.7 \%$ in 2015 , respectively). Overall, tobacco treatment/cessation programs reported owned by the hospital or its subsidiary, the health system, or other contractual mechanism increased for both NDCCs and HPOs from 2008 to 2015.

\section{Discussion}

This is the first study to our knowledge to examine tobacco treatment/cessation programs administered across U.S. hospitals providing oncology services, including both NDCCs and HPOs. The annual percentage of tobacco treatment/cessation programs provided was 
Table 1

Descriptive characteristics of hospital facilities participating in the AHA survey ${ }^{a}$ that provide oncology services, 2008-2015

\begin{tabular}{|c|c|c|c|c|c|c|c|}
\hline & & $\operatorname{NDCC}(\%)^{b}$ & & & HPO $(\%)^{c}$ & & $p^{d}$ \\
\hline Total & & 100.0 & & & 100.0 & & \\
\hline \multicolumn{8}{|l|}{$\mathrm{NCl}$ designation } \\
\hline Comprehensive Cancer Center & & 86.4 & & - & - & & \\
\hline Cancer Center & & 13.6 & & - & - & & \\
\hline \multicolumn{8}{|l|}{ Policy setting structure } \\
\hline Government, Federal & & 24.3 & & & 14.6 & & $<0.001$ \\
\hline Government, non-Federal & & 74.5 & & & 71.4 & & \\
\hline Not for profit (non-government) & & 0.0 & & & 12.3 & & \\
\hline \multirow[t]{2}{*}{ Private (for profit) } & & 1.0 & & & 1.8 & & \\
\hline & Mean & Median & Std. dev. & Mean & Median & Std. dev. & $p$ \\
\hline \multicolumn{8}{|l|}{ Capacity } \\
\hline Total licensed beds & 571 & 461 & 434 & 277 & 215 & 250 & $<0.001$ \\
\hline \multicolumn{8}{|l|}{ Utilization (annually) } \\
\hline Admissions & 23,196 & 20,128 & 17,442 & 10,736 & 8,090 & 10,615 & $<0.001$ \\
\hline Inpatient days & 138,778 & 115,793 & 109,351 & 55,557 & 39,203 & 58,765 & $<0.001$ \\
\hline Total outpatient visits & 626,075 & 441,299 & 699,173 & 229,275 & 138,925 & 267,808 & $<0.001$ \\
\hline \multicolumn{8}{|l|}{ Staffing } \\
\hline Total facility personnel & 5,553 & 4,118 & 5,555 & 1,490 & 970 & 1,712 & $<0.001$ \\
\hline
\end{tabular}

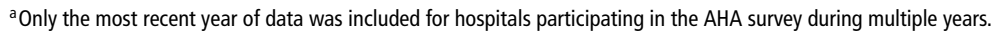

${ }^{\mathrm{b}} \mathrm{Hospital}$ directly affiliated with $\mathrm{NCl}$-Designated Cancer Centers.

'Hospitals providing oncology services.

${ }^{\mathrm{d}}$ Probability between group (NDCC and HPO) differences occurred by chance.

significantly higher among NDCCs than HPOs. The prevalence of tobacco treatment/cessation programs owned or provided by NDCCs and HPOs increased from 2008 to 2015 . NDCCs reported significantly less staffing than HPOs.

While the majority of the tobacco treatment/cessation programs were owned or provided by their hospital or its subsidiary, a smaller percentage were provided by their health systems in the local community. This is somewhat consistent with a previous study, where NDCCs reported the majority of tobacco use treatment programs being owned by their centres (59\%), and a lower percentage being external tobacco use treatment services in their health care system (21\%) (Goldstein et al., 2013). The higher reported number of total hospital staffing may further support the ability of NDCCs to provide tobacco treatment/cessation programs by the hospital as opposed to referring patients to an external, in-network resource in the local community. While the proportion of NDCCs with cessation programs and the increasing trend reported here are encouraging, a majority of cancer centers have stated that they do not perceive tobacco cessation treatment delivery as a core health care service (Goldstein et al., 2013). Therefore, the high percentage of programs available in hospitals may not reflect the actual use of these programs. One study of online resources suggested that detailed risks of tobacco use and cessation program contact information may be lacking for cancer patients (Singhi et al., 2015). Additionally, some have reported that smokers may be motivated to quit smoking following a cancer diagnosis, (Nayan, Gupta, Strychowsky, \& Sommer, 2013; Westmaas et al., 2015), but health professionals often miss opportunities to recommend smoking cessation (Earle \& Neville, 2004; Sabatino, 2007; Underwood et al., 2012; Weaver et al., 2012).

NCI Comprehensive Cancer Centers are required to develop effective cancer prevention and education approaches; however, the types of tobacco treatment/cessation programs offered and how they are administered have yet to be evaluated. NCI recently released short- and long-term initiative funding to enhance NDCC capacity to address tobacco cessation with cancer patients and implement sustainable tobacco cessation treatment programs (National Cancer Institute, 2017a). Further examination of the adoption, referral, use and barriers to use, and efficacy of cessation methods in these hospitals would inform efforts to reduce smoking among cancer survivors in hospitals and non-hospital settings. NDCCs and HPOs may also consider voluntary implementation of the Joint Commission's hospital cessation measures (The Joint Commission, 2012). The Centers for Disease Control and Prevention's (CDC) National Comprehensive Cancer Control Program (NCCCP) supports increasing knowledge and availability of evidence-based 
Table 2

Proportion of oncology service providing facilities with tobacco treatment/cessation programs, 2008-2015

\begin{tabular}{|c|c|c|c|c|c|c|c|c|c|c|}
\hline & & & $\mathrm{NDCC}^{\mathrm{a}}$ & & & & & $\mathrm{HPO}^{\mathrm{b}}$ & & \\
\hline & & & Tobacco Treatment & ssation Program & & & & Tobacco Treatment/ & sation Program & \\
\hline & Total Facilities ${ }^{\mathrm{a}}$ & Any ${ }^{c}$ & Owned by Hospital ${ }^{d}$ & Provided by System ${ }^{\mathrm{e}}$ & Other ${ }^{f}$ & Total Facilities & Any $^{c}$ & Owned by Hospital ${ }^{d}$ & Provided by System ${ }^{\mathrm{e}}$ & Other ${ }^{f}$ \\
\hline & $N$ & $n(\%)$ & $n(\%)$ & $n(\%)$ & $n(\%)$ & $N$ & $n(\%)$ & $n(\%)$ & $n(\%)$ & $n(\%)$ \\
\hline Total & 784 & $726(92.6)$ & $672(85.7)$ & $195(24.9)$ & $26(3.3)$ & 18,281 & $14,126(77.3)$ & $12,438(68.0)$ & $3,021(16.5)$ & $396(2.2)$ \\
\hline 2008 & 97 & $85(87.6)$ & $80(82.5)$ & $20(20.6)$ & $0(0.0$ & 2367 & $1,782(75.3)$ & $1,595(67.4)$ & $319(13.5)$ & $38(1.6)$ \\
\hline 2009 & 97 & $88(90.7)$ & $84(86.6)$ & $22(22.7)$ & $1(1.0)$ & 2295 & $1,723(75.1)$ & $1,543(67.2)$ & $317(13.8)$ & $39(1.7)$ \\
\hline 2010 & 96 & $89(92.7)$ & $82(85.4)$ & $24(25.0)$ & $1(1.0)$ & 2302 & $1,740(75.6)$ & $1,544(67.1)$ & $333(14.5)$ & $41(1.8)$ \\
\hline 2011 & 97 & $91(93.8)$ & $83(85.6)$ & $24(24.7)$ & $3(3.1)$ & 2314 & $1,760(76.1)$ & $1,563(67.5)$ & $344(14.9)$ & $41(1.8)$ \\
\hline 2012 & 98 & $91(92.9)$ & $84(85.7)$ & $23(23.5)$ & $3(3.1)$ & 2265 & $1,764(77.9)$ & $1,545(68.2)$ & $394(17.4)$ & $53(2.3)$ \\
\hline 2013 & 100 & $95(95.0)$ & $89(89.0)$ & $27(27.0)$ & $6(6.0)$ & 2264 & $1,782(78.7)$ & $1,542(68.1)$ & 433 (19.1) & $50(2.2)$ \\
\hline 2014 & 97 & 93 (95.9) & $86(88.7)$ & $25(25.8)$ & $6(6.2)$ & 2213 & $1,759(79.5)$ & $1,530(69.1)$ & 433 (19.6) & $60(2.7)$ \\
\hline 2015 & 102 & $94(92.2)$ & 84 (82.4) & $30(29.4)$ & $6(5.9)$ & 2261 & $1,816(80.3)$ & $1,576(69.7)$ & 448 (19.8) & $74(3.3)$ \\
\hline Trend ( $p$ ) & & 0.03 & 0.34 & 0.07 & $<0.001$ & & $<0.001$ & 0.01 & $<0.001$ & $<0.001$ \\
\hline
\end{tabular}

a Hospital directly affiliated with NCl-Designated Cancer Center.

${ }^{b}$ Hospitals providing oncology services.

'Any tobacco treatment/cessation program.

eProvided by Health System (in local community).

'Provided through a formal contractual arrangement or joint venture with another provider that is not in the hospital health system (in local community). 
tobacco cessation services among cancer survivors (Comprehensive Cancer Control National Partnership, 2016; Division of Cancer Prevention and Control Centers for Disease Control and Prevention, 2017). Partnerships between cancer coalitions, NDCCs, and HPOs may provide a unique way to identify and promote cessation services since cancer coalitions are uniquely positioned in different communities that would allow for scalability of cessation approaches beyond the hospital setting (Comprehensive Cancer Control National Partnership, 2016). NCCCP programs and coalitions working to maximise tobacco cessation behaviours among cancer survivors could identify hospitals treating cancer patients that do not offer tobacco treatment/cessation services to enable prioritising developing services for those with the most need. NCCCP programs and coalitions, in conjunction with state/local tobacco coalitions/programs, could also continue to highlight tobacco use in cancer survivors and the importance of cessation for this population.

\section{Limitations}

The study is subject to several limitations: AHA survey data are self-reported by hospitals each year; individual hospital measures reported are not completely validated; and the data are based on a sample of U.S. hospitals. However, these data are corrected for unusual changes and serve as the primary hospital-related reference for several government agencies (American Hospital Association, 2017b). AHA data do not provide details on the type, extent, or specifics of any tobacco treatment/cessation programs offered, used, or referral follow-up. Results aggregated over time include surveys from the same hospital in different years, but accurately reflect current services provided by each hospital during the year the survey was completed.

\section{Conclusions}

More than $80 \%$ of hospitals providing oncology services report providing tobacco cessation programs with higher percentages reported in NDCCs. As hospitals implement more smoking cessation programs, partnerships between hospitals and cancer coalitions could help to bring tobacco cessation activities to the communities that they both serve and link discharged patients to these cessation resources so that they can continue quit attempts that they initialised while hospitalised.

\section{Acknowledgements}

All authors were involved in conceptualising, executing, developing, and reviewing this manuscript. The contents of this article have not been previously presented elsewhere.

\section{Financial Support}

All funding was provided by the Centers for Disease Control and Prevention.

\section{Conflict of Interest}

The authors have no further conflicts of interest to declare.

\section{Ethical Standards}

The authors assert that all procedures contributing to this work comply with the ethical standards of the relevant national and institutional committees on human experimentation and with the Helsinki Declaration of 1975, as revised in 2008. The findings and conclusions in this report are those of the authors and do not necessarily represent the official position of the Centers for Disease Control and Prevention.

\section{References}

American Hospital Association (2017a). 2015 AHA annual survey. Retrieved July 8, 2017 from https: //www.ahadataviewer.com/Global/survey\%20instruments/ 2015AHAAnnualsurvey.pdf

American Hospital Association (2017b). American Hospital Association annual survey database. Retrieved from https:// www.ahadataviewer.com/about/data/

Blum, A. (1997). Cancer prevention: Preventing tobacco-related cancers. In: DeVita V.T., Jr, Hellman S \& Rosenberg SA (Eds.), Cancer: Principles and practice of oncology. Ed 5 Lippincott-Raven, Philadelphia, 545-557.

Browman, G. P., Wong, G., Hodson, I., Sathya, J., Russell, R., McAlpine, L. et al. (1993). Influence of cigarette smoking on the efficacy of radiation therapy in head and neck cancer. The New England Journal of Medicine, 328(3), 159-163. doi:10.1056/NEJM199301213280302

Comprehensive Cancer Control National Partnership (2016). Increase availability of tobacco cessation services for cancer survivors. Retrieved from http://www. cccnationalpartners.org/increase-availability-tobaccocessation-services-cancer-survivors

Cox, L., Africano, N., Tercyak, K., \& Taylor, K. (2003). Nicotine dependence treatment for patients with cancer. Cancer, 98(3), 632-644.

Day, G. L. (1994). Second cancers following oral and pharyngeal cancers: Role of tobacco and alcohol. Journal of the National Cancer Institute, 86(2), 131-137.

Des Rochers, C., Dische, S., \& Saunders, M. I. (1992). The problem of cigarette smoking in radiotherapy for cancer in the head and neck. Clinical Oncology, 4(4), 214-216.

Division of Cancer Prevention and Control Centers for Disease Control and Prevention (2017). National Comprehensive Cancer Control Program (NCCCP). Retrieved March 7, 2017 from https://www.cdc.gov/cancer/nccep/

Do, K. A., Johnson, M., Doherty, D., Lee, J. J., Wu, X., Dong, Q. et al. (2003). Second primary tumors in patients with upper aerodigestive tract cancers: Joint effects of smoking and alcohol (United States). Cancer Causes \& Control, 14(2), 131138.

Earle, C. C., \& Neville, B. (2004). Under use of necessary care among cancer survivors. Cancer, 101(8), 1712-1719. 
Emmons, K., Li, F., Whitton, J., Mertens, A., Hutchinson, R., Diller, L. et al. (2002). Predictors of smoking initiation and cessation among childhood cancer survivors: A report from the childhood cancer survivor study. Journal of Clinical Oncology, 20(6), 1608-1616.

Fiore, M. C., Jaen, C. R., Baker, T. B., Bailey, W. C., Benowitz, N. L., Curry, S. J. et al. (2008). Treating tobacco use and dependence: 2008 update. Clinical practice guideline. Rockville, Maryland: US Department of Health and Human Services, Public Health Service.

Gan, S. J., Dahlstrom, K., Peck, B., Caywood, W., Li, G., Wei, Q. et al. (2013). Incidence and pattern of second primary malignancies in patients with index oropharyngeal cancers versus index nonoropharyngeal head and neck cancers. Cancer, 119(14), 2593-2601.

Goldstein, A. O., Ripley-Moffitt, C. E., Pathman, D. E., \& Patsakham, K. M. (2013). Tobacco use treatment at the U.S. national cancer institute's designated cancer centers. Nicotine \& Tobacco Research, 15(1), 52-58.

Gritz, E. R., Fingeret, M., Vidrine, D., Lazev, A., Mehta, N., \& Reece, G. (2006). Successes and failures of the teachable moment. Cancer, 106(1), 17-27.

Gritz, E. (2005). Smoking, the missing drug interaction in clinical trials: Ignoring the obvious. Cancer Epidemiology, Biomarkers \& Prevention, 14(10), 2287-2293.

Kawahara, M., Ushijima, S., Kamimori, T., Kodama, N., Ogawara, M., Matsui, K. et al. (1998). Second primary tumours in more than 2-year disease-free survivors of smallcell lung cancer in Japan: The role of smoking cessation. British Journal of Cancer, 78(3), 409-412.

Khuri, F. R., Lee, J. J., Lippman, S. M., Kim, E. S., Cooper, J.S., Benner, S. E. et al. (2006). Randomized phase III trial of low-dose isotretinoin for prevention of second primary tumors in stage I and II head and neck cancer patients. Journal of the National Cancer Institute, 98(7), $441-450$.

Klosky, J. L., Tyc, V., Garces Webb, D., Buscemi, J., Klesges, R., \& Hudson, M. (2007). Emerging issues in smoking among adolescent and adult cancer survivors. Cancer, 110(11), 2408-2419.

Mackenbach, J. P. (2001). Determinants of levels and changes of physical functioning in chronically ill persons: Results from the GLOBE Study. Journal of Epidemiology and Community Health, 55(9), 631-638.

Mariotto, A. (2007). Multiple cancer prevalence: A growing challenge in long-term survivorship. Cancer Epidemiology, Biomarkers \& Prevention, 16(3), 566-571.

Mason, D. P., Subramanian, S., Nowicki, E., Grab, J., Murthy, S., Rice, T. et al. (2009). Impact of smoking cessation before resection of lung cancer: A society of thoracic surgeons general thoracic surgery database study. The Annals of Thoracic Surgery, 88(2), 362-371.

National Cancer Institute (2017a). Comprehensive Cancer Center Cessation Initiative (P30 supplement). Retrieved from https://www.cancer.gov/about-nci/ organization/crchd/funding-training-opportunities/ adminsupplement-non-fy15
National Cancer Institute (2017b). NCI-Designated Cancer Centers. Retrieved from https://www.cancer.gov/research/ nci-role/cancer-centers

Nayan, S., Gupta, M. K., Strychowsky, J. E., \& Sommer, D. D. (2013). Smoking cessation interventions and cessation rates in the oncology population: An updated systematic review and meta-analysis. Otolaryngology - Head and Neck Surgery, 149(2), 200-211. doi:10.1177/0194599813490886

Office of Disease Prevention and Health Promotion (2017). Healthy people 2020, tobacco use objectives. Retrieved from https://www.healthypeople.gov/2020/topics-objectives/ topic/tobacco-use/objectives

Ramaswamy, A. T., Toll, B., Chagpar, A., \& Judson, B. (2016). Smoking, cessation, and cessation counseling in patients with cancer: A population-based analysis. Cancer, 122(8), 1247-1253.

Richardson, G. E. (1993). Smoking cessation after successful treatment of small-cell lung cancer is associated with fewer smoking-related second primary cancers. Annals of Internal Medicine, 119(5), 383-390.

Sabatino, S. (2007). Provider counseling about health behaviors among cancer survivors in the United States. Journal of Clinical Oncology, 25(15), 2100-2106.

Shiels, M. S., Gibson, T., Sampson, J., Alabanes, D., Andreotti, G., Freeman, L. B. et al. (2014). Cigarette smoking prior to first cancer and risk of second smoking-associated cancers among survivors of bladder, kidney, head and neck, and stage I lung cancers. Journal of Clinical Oncology, 32(35), 3989-3995. doi:10.1200/JCO.2014.56.8220

Shoemaker, M., White, M., Hawkins, N., \& Hayes, N. (2016). Prevalence of smoking and obesity among U.S. cancer survivors: Estimates from the national health interview survey, 2008-2012. Oncology Nursing Forum, 43(4), 436-441.

Singhi, E. K., Pommerenke, A. W., Mushtaq, S., Cummings, K. M., Marshall, J. R., Alberg, A. et al. (2015). Addressing tobacco use at NCI designated cancer centers: Online resources available for patients. Journal of Clinical Oncology, 33(15_Suppl), e12566-e12566. doi:10.1200/jco.2015.33.15_suppl.e12566

Siu, A. L. (2015). Behavioral and pharmacotherapy interventions for tobacco smoking cessation in adults, including pregnant women: U.S. preventive services task force recommendation statement. Annals of Internal Medicine, 163(8), 622-634.

The Joint Commission (2012). Tobacco treatment measures. Retrieved from http://www.jointcommission.org/assets/1/6/ Tobacco\%20Treatment\%20Measures\%20List1.PDF

Tucker, M. A. (1997). Second primary cancers related to smoking and treatment of small-cell lung cancer. Lung cancer working cadre. Journal of the National Cancer Institute, 89(23), 1782-1788.

Tyc, V. L. (2005). A comparison of tobacco-related risk factors between adolescents with and without cancer. Journal of Pediatric Psychology, 30(4), 359-370.

Underwood, J. M., Townsend, J., Stewart, S. L., Buchannan, N., Ekwueme, D. U., Hawkins, N. A. et al. (2012). Surveillance 
of demographic characteristics and health behaviors among adult cancer survivors - behavioral risk factor surveillance system, united states, 2009. Morbidity and Mortality Weekly Report, 61(1), 1-23.

Underwood, J. M., Townsend, J., Tai, E., White, A., Davis, S., \& Fairley, T. (2012). Persistent cigarette smoking and other tobacco use after a tobacco-related cancer diagnosis. Journal of Cancer Survivorship, 6(3), 333-344.

Weaver, K. E., Danhauer, S. C., Tooze, J. A., Blackstock, A. W., Spangler, J., Thomas, L. et al. (2012). Smoking cessation counseling beliefs and behaviors of outpatient oncology providers. The oncologist, 17(3), 455-462.

Westmaas, J. L., Newton, C. C., Stevens, V. L., Flanders, W. D., Gapstur, S. M., \& Jacobs, E. J. (2015). Does a recent cancer diagnosis predict smoking cessation? An analysis from a large prospective US cohort. J Clin Oncol, 33(15), 16471652. doi:10.1200/JCO.2014.58.3088

Wynder, E. (1977). Tobacco and alcohol consumption in relation to the development of multiple primary cancers. Cancer, 40(S4), 1872-1878. 